\title{
Endomyocardial complications of the Churg-Strauss syndrome
}

\author{
J.G. Lanham, S. Cooke ${ }^{1}$, J. Davies and G.R.V. Hughes \\ Department of Medicine, Hammersmith Hospital and Royal Postgraduate Medical School, London, and ${ }^{\prime}$ Kingston \\ Hospital, Kingston-upon-Thames, UK.
}

\begin{abstract}
Summary: Although many similarities exist between the Churg-Strauss syndrome and the hypereosinophilic syndrome, these two disorders have hitherto been characterized by different types of cardiac disease. Two cases of the Churg-Strauss syndrome are described where the typical endomyocardial lesion of the hypereosinophilic syndrome dominated the clinical picture.
\end{abstract}

\section{Introduction}

Hypereosinophilic syndrome (HES) and ChurgStrauss syndrome (CSS) are similar multi-system disorders falling within the disease spectrum described by Engelfeldt \& Zetterstrom (1956) as the 'Disseminated Eosinophilic Collagen Diseases.' The two syndromes can usually be distinguished on clinical and pathological grounds, although both are characterized by the accumulation of large numbers of eosinophils in the tissues and blood. A necrotizing vasculitis is an important component of CSS (Churg \& Strauss, 1951), and granulomas are found in a proportion of cases (Chumbley et al., 1977; Lanham et al., 1984). The clinical picture of CSS is most distinctive: the initial phase is marked by asthma and allergic rhinitis with nasal polyposis; this is often followed by an eosinophilic infiltrative phase mimicking Loeffler's syndrome or eosinophilic gastroenteritis, but ultimately a life-threatening vasculitic illness intervenes (Lanham et al., 1984). On the other hand, asthma is seldom a feature of HES (Spry, 1982), and this syndrome has no vasculitic component. The disorder is dominated by cardiac and cerebral involvement, and thromboembolic complications are common (Spry et al., 1983). The latter phenomenon probably accounts for the renal disease of HES, while a focal segmental glomerulonephritis is typical of CSS (Lanham et al., 1984). The sex incidence is almost equal in CSS, whereas HES is 9 times more common in males.

J.G. Lanham, M.B., B.Ch., M.R.C.P.; S. Cooke, B.Sc., M.B., B.S., M.R.C.P.; J. Davies, M.D., M.R.C.P.; G.R.V. Hughes, M.D., F.R.C.P.

Correspondence: J.G. Lanham, Department of Rheumatology, St Bartholomew's Hospital, London EC1A $7 B E$.

Accepted: 20 June 1984
The heart is a primary target organ in both syndromes. In CSS, pericardial and myocardial involvement manifests as acute or constrictive pericarditis, cardiac failure or myocardial infarction (Davidson et al., 1983; Lanham et al., 1984). With one possible exception (Case records of the Massachusett's General Hospital, 1980), the eosinophilic endomyocarditis typical of HES has not previously been recognized in living patients with CSS. This form of cardiac disease progresses from an early eosinophilic necrotic phase to fibrosis, producing a picture resembling endomyocardial fibrosis (Davies et al., 1983). We report two patients with CSS who developed the cardiac complications of HES.

\section{Case reports}

\section{Case 1}

A white woman developed asthma and allergic rhinitis at the age of 40 , having had no previous personal or family history of atopy. Two years later she presented with severe muscle cramps in the calves, and was found to have peripheral blood eosinophilia and repolarisation abnormalities on her electrocardiogram (ECG). This episode occurred in early spring as had previous asthmatic attacks, and settled spontaneously within a few months. The following spring, she again developed asthma and leg cramps, and became systemically unwell with fever and marked weight loss. A left popliteal nerve palsy developed, and hypertension was noted. No skin lesions were present apart from splinter haemorrhages; examination of the cardiovascular system was normal. Nevertheless her heart size was found to be increasing radiologically and a

(C) The Fellowship of Postgraduate Medicine, 1985 
pericardial effusion was present on echocardiogram. The eosinophil count was $4.7 \times 10^{9} / 1 ; 3 \%$ of the eosinophils were vacuolated and $1 \%$ were degranulated. Haematuria and an active urinary sediment was found, but renal function was not impaired. Visceral angiography was normal, and renal biopsy showed a necrotizing segmental glomerulonephritis; vasculitis with fibrinoid necrosis was noted in a large vessel, and an eosinophilic interstitial infiltrate was present. Churg-Strauss syndrome was diagnosed and treatment commenced with prednisolone $40 \mathrm{mg}$ daily with rapid suppression of the peripheral blood eosinophilia. On reduction of the steroid dose she experienced malaise and arthralgias, and azathioprine was introduced.

Shortly thereafter she complained of coldness and discolouration of her left foot; distal pulses were absent in the left leg and she was thought to have had an embolus. In the ensuing 9 months the prednisolone dose was gradually reduced to $5 \mathrm{mg}$ daily without recurrence of vasculitis or asthma; hypertension was controlled with atenolol $200 \mathrm{mg}$ and hydralazine $200 \mathrm{mg} / \mathrm{d}$. She then developed ankle oedema and her jugular venous pressure was found to be raised at $8 \mathrm{~cm}$ with a positive Kussmaul's sign; a third heart sound was noted. The heart size was slightly reduced radiologically, and no pericardial effusion was detectable on echocardiography. Constrictive pericarditis was diagnosed and she initially responded to treatment with frusemide $40 \mathrm{mg}$ daily. She then developed recurrent vomiting, and was found to be in severe right ventricular failure with a soft pansystolic murmur at the left sternal border. Peripheral perfusion was

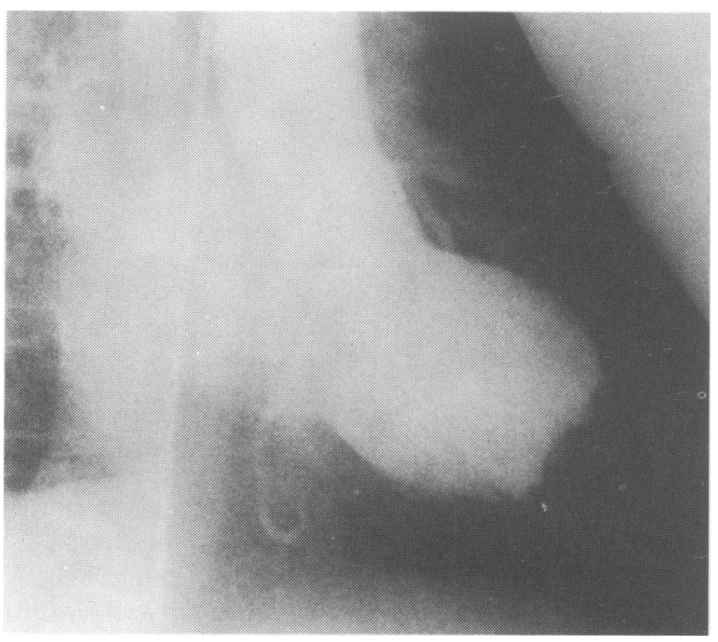

Figure 1 Left ventricular angiogram showing small well-contracting ventricular cavity with gross obliteration of the left ventricular apex (Case 1).

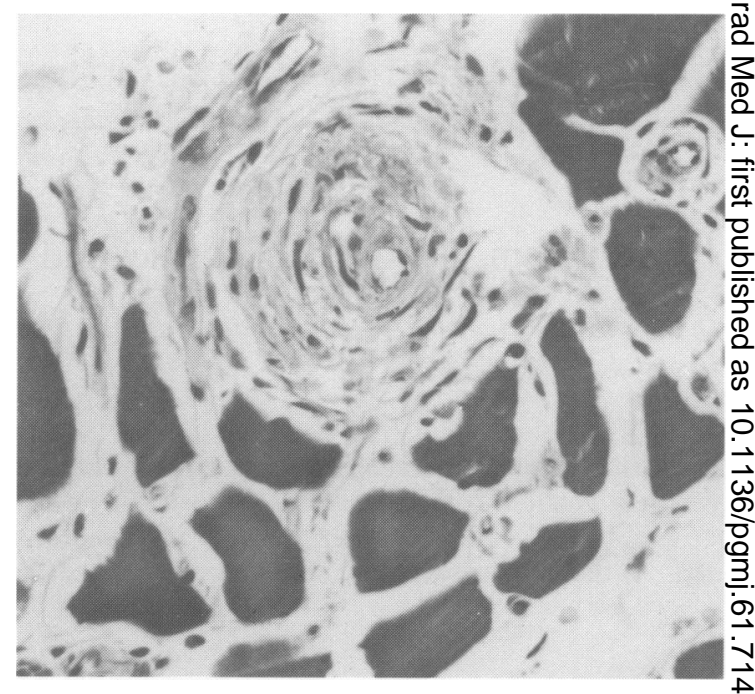

Figure 2 Muscle biopsy showing necrotizing vasculitis in a small artery (Case 2).

generally poor. Cardiac catheterization showed ad- $\frac{7}{3}$ vanced restrictive cardiomyopathy with obliteration of the apices of both ventricles in the pattern typical $\mathscr{O} \mathscr{C}_{\infty}$ endomyocardial fibrosis (Figure 1). An emergen e or laparotomy was performed because of abdomin pain. A large mesenteric artery embolus was foun and resection of small and large bowel was required.

Post-operatively renal function deteriorated and she suffered a right sided hemiplegia and died. Autopsy $\underset{\square}{\mathbb{Q}}$ was not permitted.

\section{Case 2}

A 61 year old white man first developed asthma 103 years ago following an illness characterized by pul- $\dot{0}$ monary infiltrates and peripheral blood eosinophilia. Since then his asthma had been mild, but he presented $\delta$ with a 5 week history of myalgias affecting the calves, a rash on the lower legs and exertional dyspnoea with 음 peripheral oedema. He had also noted parasthesiae in the left hand, and had lost 10 pounds in weight. On examination he was febrile and had a purpuric rash on the legs. He was normotensive with a tachycardia of $\infty$ 108 beats/min and his jugular venous pressure was $\tilde{O}$ raised $10 \mathrm{~cm}$. There was no clinical evidence of cardiomegaly, but a right ventricular fourth heart sound 0 and a pericardial rub were audible. His liver was palpable $2 \mathrm{~cm}$ below the costal margin and bilateral leg oedema was present.

Investigations revealed a haemoglobin of $11.7 \mathrm{~g} / 1 ; \mathrm{a}$ white blood count of $11.2 \times 10^{9} / 1$ with $5.6 \times 10^{9} / 1$ eosinophils. Platelet count was normal. Erythrocyte $\stackrel{\square}{\square}$ 
sedimentation rate was $50 \mathrm{~mm}$ in the first hour, alkaline phosphatase was raised $(202 \mathrm{IU} / 1)$ and tests of liver and renal function were normal. The chest radiograph was normal and the ECG showed T-wave inversion in inferior and lateral leads. Muscle biopsy showed a necrotizing vasculitis (Figure 2). Echocardiography demonstrated partial obliteration of the right ventricular cavity with multiple layers of thrombus. Angiography confirmed this finding and also showed early obliteration of the left ventricular cavity. Both mitral and tricuspid regurgitation were documented. A right ventricular biopsy revealed marked interstitial fibrosis with muscle hypertrophy, but no eosinophilic infiltrate or vasculitis (Figure 3). Left ventricular biopsy was normal. While being investigated he developed a mononeuritis affecting the right median nerve but after treatment with prednisolone $40 \mathrm{mg}$ daily, warfarin and a thiazide diuretic his disease remitted and he has subsequently remained free of symptoms.

\section{Discussion}

Both cases have histologically proven necrotizing vasculitis, as well as asthma and peripheral blood eosinophilia. These features together with allergic rhinitis, focal segmental glomerulonephritis and pulmonary infiltrates are all typical of CSS. However, their cardiac lesions are characteristic of HES. This type of heart disease is an obliterative and restrictive cardiomyopathy (Davies et al., 1983). Acute myocardial necrosis is evident in the early lesions and later microvascular fibrin deposition develops, often with extensive overlying thrombus. The process typically affects the inflow tracts of both ventricles, and can obliterate much of the ventricular cavity. Tethering of the chordae tendinae may produce tricuspid and mitral regurgitation (Parillo et al., 1979). The advanced cardiac lesion of HES resembles endomyocardial fibrosis, yet the latter condition is not associated with eosinophilia. The enigmatic relationship between these two disorders has recently been clarified by a report on the late development of endomyocardial fibrosis in 13 of 44 patients treated for microfilariasis associated with eosinophilia (Andy et al., 1981). In these cases, as in case 1 , the cardiac lesion appeared months after the eosinophilic illness and the peripheral blood eosinophil count was not raised when the cardiac disease presented.

This cardiac lesion has also been reported in association with a variety of other allergic, neoplastic and parasitic disorders which are accompanied by hypereosinophilia. Thus, a raised peripheral blood eosinophil count is a factor common to a range of

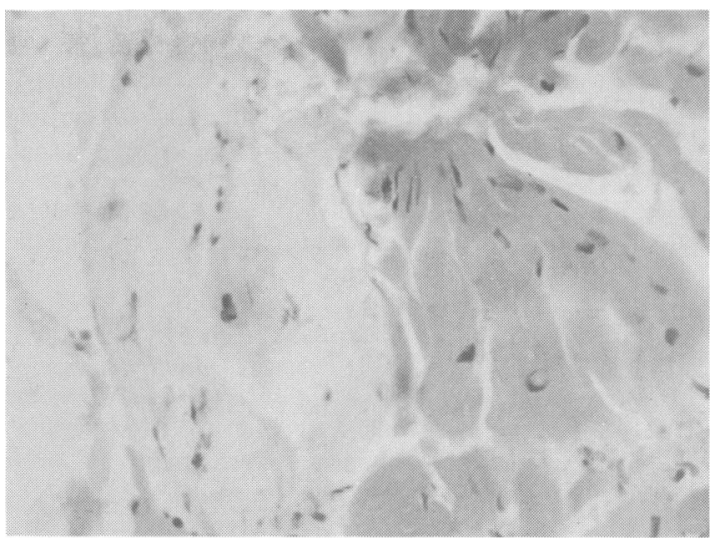

Figure 3 Right ventricular biopsy from case 2 showing interstitial fibrosis with hypertrophy of adjacent cardiac muscle cells. (H. \& E. $\times 400)$

disparate conditions complicated by this type of heart disease. The role of the eosinophil in the induction of cardiac damage is yet to be established, but evidence of eosinophil degranulation is often seen (Spry, 1982). The release of eosinophil myeloperoxidase, major basic protein, cationic protein and Charcot Leyden crystal protein may be responsible for some of the clinical features of the disorder (Spry, 1982).

These two cases underline the similarities between CSS and HES. HES is not a distinct disease entity but a heterogenous group of disorders, which range from disease limited to skin rash and hypereosinophilia to that with haematological and cytogenetic features of a true eosinophilic leukaemia (Spry, 1982). Patients with pulmonary disease, angio-oedema, high IgE levels and hypereosinophilia, constitute another sub-group and their disease tends to respond well to treatment with steroids (Bush et al., 1978).

Other patients have more pronounced cardiac and cerebral involvement, and a poorer prognosis. CSS, on the other hand resembles not only HES, but also vasculitic disorders such as polyarteritis nodosa and granulomatous conditions such as Wegener's granulomatosis. Our cases emphasize the value of viewing these syndromes as a clinicopathological continuum, rather than as independent disorders.

\section{Acknowledgements}

We thank Dr M. Webb-Peploe for performing the cardiac investigations in Case 2, and Dr N.C. Medd for allowing us to report this case. 


\section{References}

ANDY, J.J., BISHARA, F.F. \& SOYINKA, O.O. (1981). Relation of severe eosinophilia and microfilariasis to chronic African endomyocardial fibrosis. British Heart Journal, $45,672$.

BUSH, R.K., GELLER, M., BUSSE,W.W., FLAHERTY, D.K. \& DICKIE, H.A. (1978). Response to corticosteroid in the hypereosinophilic syndrome. Archives of Internal Medicine, 138, 1244.

CASE RECORDS OF THE MASSACHUSETTS GENERAL HOSPITAL (1980). Case 18. New England Journal of Medicine, 302, 1077.

CHUMBLEY, L.C., HARRISON, E.G. \& DE REMEE, R.A. (1977). Allergic granulomatosis and angiitis (ChurgStrauss Syndrome). Mayo Clinic Proceedings, 52, 477.

CHURG, J. \& STRAUSS, L. (1951). Allergic granulomatosis, allergic angiitis and periarteritis nodosa. American Journal of Pathology, 27, 277.

DAVIES, J., SPRY, C.J.F., SAPSFORD, R., OLSEN, E.G.J., DE PEREZ, G., OAKLEY, C.M. \& GOODWIN, J.F. (1983). Cardiovascular features of 11 patients with eosinophilic endomyocardial disease. Quarterly Journal of Medicine, $52,23$.
DAVIDSON, A.G., THOMPSON,P.J., DAVIES, J., CORRIN, B.\& TURNER-WARWICK, M. (1983). Prominent pericardial and myocardial lesions in the Churg-Strauss syndrome (allergic granulomatosis and angiitis). Thorax, 38, 793.

ENGELFELDT, B. \& ZETTERSTROM, R. (1956). Disseminated eosinophilic collagen disease. Acta Medica Scandinavica, 153, 337.

LANHAM, J.G., ELKON, K.B., PUSEY, C.D. \& HUGHES, G.R.V. (1984). Systemic vasculitis with asthma and eosinophilia: a clinical approach to the Churg-Strauss syndrome. Medicine, 63, 65.

PARILLO, J.E., BORER, J.S., HENRY, W.L., WOLFF, S.M. \& FAUCI, A.S. (1979). The cardiovascular manifestations of the hypereosinophilic syndrome. American Journal of Medicine, 67, 572.

SPRY, C.J.F. (1982). The hypereosinophilic syndrome: clinical features, laboratory findings and treatment. Allergy, 37, 539.

SPRY, C.J.F., DAVIES, J., TAI, P.C., OLSEN, E.G.J., OAKLEY, C.M. \& GOODWIN, J.F. (1983). Clinical features of fifteen patients with hypereosinophilic syndrome. Quarterly Journal of Medicine, 52, 1. 